\title{
Mycobacterium Avium Complex (MAC) Lung Disease in Two Inner City Community Hospitals: Recognition, Prevalence, Co-Infection with Mycobacterium Tuberculosis (MTB) and Pulmonary Function (PF) Improvements After Treatment
}

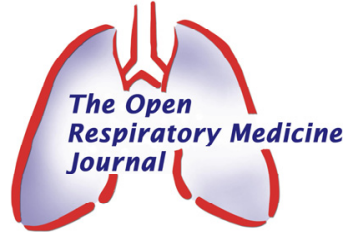

\author{
Zinobia Khan*, Albert Miller, Moses Bachan and Joseph Donath
}

\author{
CARITAS Health Care Inc, Mary Immaculate Hospital-Pulmonary Division, 152-11 $89^{\text {th }}$ Ave, Jamaica, New York \\ 11432, USA
}

\begin{abstract}
Introduction: The purpose of this study was to separate MAC lung disease from colonization and to define indications for treatment.

Materials and Methodology: Over 4 years, we evaluated patients who had positive MAC cultures, MAC infection and coinfection with MTB. In the first study, 42 immunocompetent patients with sputum or BAL culture positive only for MAC during a single year (2004) were reviewed. On clinical and radiographic review, they were classified as disease related to MAC, likely related to MAC or unrelated to MAC. In the second study, we reviewed all immunocompetent patients, during two years (2004-2005), whose respiratory secretions cultured both MTB and nontuberculous mycobacteria (NTM). In the last study, we evaluated pulmonary function (PF) in patients with MAC infection before and after therapy (20062007). PF was evaluated in patients following ATS guidelines.

Results: Lung disease was related/likely related to MAC in 21 patients $(50 \%)$ and not related in $21(50 \%)$. In patients with MAC-related lung disease, the primary physician did not consider the diagnosis except when that physician was a pulmonologist. Half of those with MAC-related lung disease were smokers, white and US-born. There were 12 immunocompetent patients with MTB and NTM cultures. Eleven were non-white and all were foreign-born. Presentation and clinical course were consistent with MTB. All 8 patients with abnormal PF improved.

Conclusions: The prevalence of MAC lung infection in two inner city hospitals was four times higher than that of TB. The indication for treatment of MAC infection should also rely heavily on clinical and radiological evidence when there is only one positive sputum culture. The diagnosis was considered only when the admitting physician was a pulmonologist. Most patients with combined infection were clinically consistent with MTB and responded to anti MTB treatment alone. Treatment with anti-MAC therapy improved PF in those patients whose PF was abnormal to begin with.
\end{abstract}

Keywords: Mycobacterium avium complex (MAC), Mycobacterium tuberculosis (MTB), Non-Tuberculous mycobacteria (NTM), immunocompetent, bronchoalveolar lavage (BAL), bronchiectasis, cavitation, Bacillus Calmette-Guerin (BCG) vaccination, pulmonary function $(\mathrm{PF})$.

\section{INTRODUCTION}

MAC is a ubiquitous environmental microorganism. It is found in water, soil and even in cigarette components including tobacco, filter and paper [1]. It can be a laboratory contaminant, causing sporadic positive cultures.

This organism can cause disease in both immunocompetent and immunocompromised persons. In recent years, there has been an increase in disease incidence; it is not clear whether this reflects a real increase in infections or greater recognition resulting from more widespread awareness and more sensitive laboratory techniques [2].

*Address correspondence to this author at the James J. Peters VA Medical Center, 130 W. Kingsbridge Road, Bronx, New York, NY 10468, USA; Tel: (718) 496-9531, (718) 496-9854; Fax: 718-741-4623;

E-mail: zkhan36@aol.com
Lung disease caused by MAC was first described in sanatorium patients with suspected tuberculosis [3]. Before 1989, MAC disease was thought to be limited to elderly men with a history of alcohol and tobacco use and pre-existing lung disease. In 1989, Prince et al. found that fibronodular bronchiectasis was common in MAC infection of the lung, often seen in elderly women without underlying lung disease or immunosuppression [4]. In women, MAC infection mainly affects the middle lobe and lingula. In men, it affects the upper lobes, which may show fibrocavitary disease.

Studies by Beck, 1959 and Warring, 1968 showed that MAC infection may be present for months or years before diagnosis is made $[5,6]$. Some patients may be asymptomatic and come to diagnosis as an incidental x-ray finding. Many patients have chronic productive cough often attributed to COPD but usually without hemoptysis, fever, night sweats, weight loss, malaise, lethargy and fatigue unless there is extensive lung disease [7]. 
Mycobacterium Tuberculosis (MTB) and NonTuberculous Mycobacteria (NTM) lung infection often resemble each other. We were unaware of how often these organisms co-exist in our institution.

Physicians are often conflicted whether to treat patients with MAC since symptoms are often minimal or nonspecific and treatment is prolonged and associated with side effects. Many of these patients have abnormal pulmonary function $(\mathrm{PF})$, which can precede or result from MAC infection. Expected improvements in PF would be an indication to treat.

The purpose of our study is to evaluate the prevalence and nature of MAC infection, MAC co-infection with MTB and pulmonary function before and after treatment, in Mary Immaculate and Saint John's hospitals, two inner city hospitals, in Queens, New York. These hospitals served a diverse patient population including a majority of foreignborn, black, Asian and Hispanic patients. Most of these patients were generally on Medicaid or lacking insurances.

\section{MATERIALS AND METHODOLOGY}

For the studies, we reviewed medical and mycobacteriology laboratory records, chest x-rays and/or computer tomography (CT) scans, Department of Health Registry and Pulmonary Function Laboratory data from 2004 to 2007. The initial purpose of the study was to assess the incidence and likelihood of diagnosis of MAC-related lung disease, in immunocompetent and in immunocompromised patients, in two inner city hospitals, and compare this incidence with that of tuberculosis and of co-infection. As an outgrowth of these efforts, we noted that pulmonary function was impaired in many patients and questioned whether treating MAC would improve function.

For the first part of this study, all patients' whose respiratory secretions grew MAC during the year 2004 were evaluated. Immunocompromised patients and those who had active tuberculosis were excluded. Each case was classified as having lung disease (a) related, (b) likely related, or (c) unrelated to MAC infection. The patients were categorized based on the presence or absence of :(1) Clinical evidence of a chronic disease characterized by otherwise unexplained cough, sputum, weight loss and/ or fever; (2) otherwise unexplained radiographic findings including peripheral nodules with or without cavitation and bronchiectasis; (3) lack of response to standard respiratory antibiotics; (4) appropriate response to antibiotic regimen for MAC.

For the second part of this study, we evaluated all immunocompetent patients whose respiratory secretions cultured positive for either or both MTB and NTM during 2004 and 2005.

In the third part of this study, we reviewed the medical records of 10 patients who received standard MAC treatment, from 2006-2007. Nine patients completed at least 12 months of anti-MAC therapy and one patient who was unable to tolerate therapy completed 5 months. Treatment followed ATS guidelines [7]. Spirometry pre and post bronchodilator (BD) was obtained for all patients. Lung volumes and DLCO were obtained for 5 patients. Percent predicted values for FEV1 and FVC were used to monitor changes. The largest values for FEV1, FVC and FEV1/FVC either pre or post BD were used. Miller's predicted values were used $[8,9]$. The student's t-test was used for statistical analysis.

\section{RESULTS}

For the first study, at least one respiratory culture was positive for MAC in 89 patients. Ten cases were excluded because of insufficient clinical information. Another 32 patients were immunocompromised due to malignant disease, HIV positivity (29), or treatment with steroids or cytostatic drugs. Another 5 patients were excluded for coexisting active pulmonary tuberculosis. The remaining 42 patients were the subjects of detailed clinical-radiological review. Fig. (1) summarizes these results.

Thirteen patients (31\%) had disease related to MAC based on typical clinical and radiographic features: bronchiectasis in 2 patients, cavitary disease in 1 patient, nodules in 3 patients and diffuse densities in 3 patients; 4 patients had a combination of bronchiectasis, infiltrate and nodules. Sex distribution was almost equal; age ranged from 48 to 77 years. In this group, $77 \%$ had pre-existing lung disease and $46 \%$ smoked. 2 patients had only one MAC positive sputum; one had only 1 sample sent while the other had 2 samples sent. The possibility of MAC related lung disease was never considered by the primary attending physician except when that physician was a pulmonologist; pulmonary or Infectious Disease consultation was not sought. Three of the 13 patients, whose private medical doctor (PMD) were pulmonologists, diagnosis were confirmed with response to appropriate anti-MAC treatment started on admission. Three patients were treated, in the pulmonary clinics after discharge. Six patients were followup at other institutions/offices and one patient die before treatment with anti-MAC was started. These 7 patients had consistent clinical and radiographic findings for MAC lung disease. All patients had monthly sputum culture for MAC while on treatment. Figs. $(\mathbf{2}, \mathbf{3})$ shows the CT scans of two of the patients who had lung disease related to MAC.

There were eight (8) patients (19\%) who had lung abnormalities likely related to MAC. Confirmation could not be obtained since these patients were lost to long-term follow up. Of these, 4 patients had only one sputum MAC positive (only one sputum sample was sent for all 4 patients).

\section{LUNG DISEASE NOT RELATED TO MAC (21 PATIENTS)}

Twenty-one patients (50\%) who had one or more cultures positive for MAC had a lung disease clearly not related to MAC. Most of these patients were admitted for typical clinical and radiographic findings of community acquired pneumonia that resolved rapidly. In this group, 10 patients had only one sputum MAC positive (3 patients had 1 of 3 sputum samples and 7 had only one sputum sample sent). One patient had 5 sputum samples positive for MAC.

For the second study, on co-infection with MTB, thirtyone (31) patients were positive for MTB alone of whom 11 were immunocompetent; 92 immunocompetent patients were positive for MAC alone. Eighteen (18) patients were positive for both MTB and NTM, 12 of whom were immunocompetent. Eight of these twelve patients were positive for MTB and MAC. The other four patients were 
89 Patients with sputum or BAL MAC+

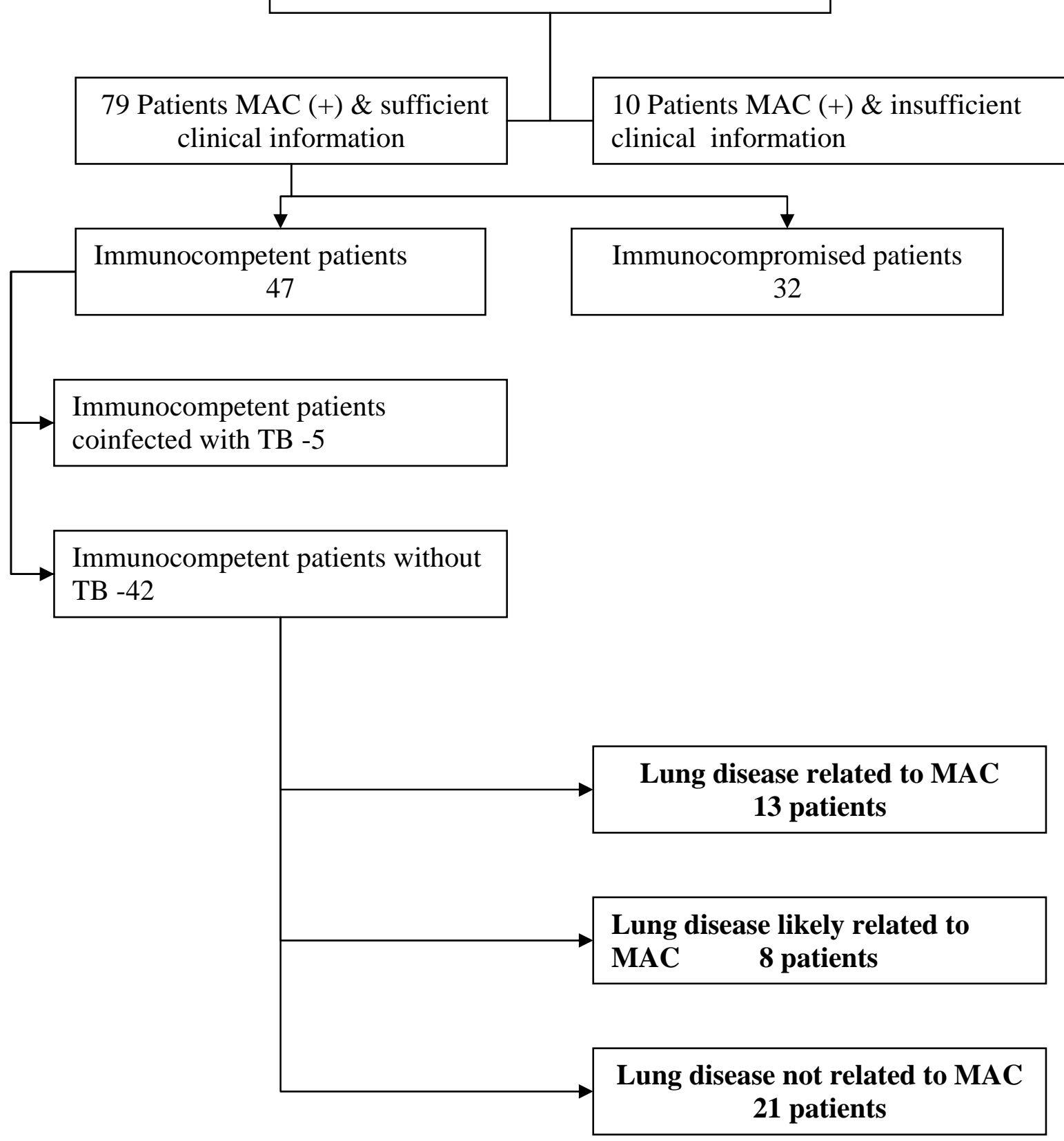

Fig. (1). Summary of immune states and MAC presentation. MAC- Mycobacterium Avium Complex, BAL-Bronchoalveolar Lavage, TBtuberculosis.

positive for MTB and other non NTM. Of the twelve dualinfected patients, age range was 20-68 years. Eight were men, 8 were smokers and 8 patients did not have any previous diseases. Their length of hospital stay varied from 6 to 50 days. Eleven were non- white and all patients were foreign born. Their presentation and clinical course were consistent with MTB. Four patients had consolidated lobes or segments. Ten patients were treated for MTB alone, one was treated for MTB and MAC. Nine of them improved over 6 months, 1 was lost to follow-up and 2 patients died of unrelated causes.
For the PF study (see Table 1). seven of the ten patients were men, six were ex-smokers and four were lifetime nonsmokers. Their age range was 49 to 81 years old and BMI range 21 to 29 . At the time of post-treatment PFTs the patients had negative sputum cultures for MAC. All the patients were followed for negative cultures on a monthly basis. For FEV1, 9 patients had increases from 2 to $17 \%$ or no change; 1 patient had a decrease of $9 \%$. For FVC, 7 patients had increases from 4 to $17 \%, 3$ had a decrease from 3 to $11 \%$. For FEV1/FVC, 7 patients had increases from 5 to $13 \%$ or no change, 3 had a decrease, $1 \%$ to $3 \%$; included in 
these three was the patient who did not complete therapy. For 3 patients, DLCO increased by $4 \%$ to $27 \%$ (mean change, $16 \%$ ) and in 2 patients it decreased by $5 \%$ and $8 \%$, (mean change, 7\%). TLC increased in all 5 patients tested, by $3 \%$ to $9 \%$. In 3 patients, FRC $\%$ decreased by $9 \%, 21 \%$ and $25 \%$ (mean change, $18 \%$ ) and in 2 patients it increased by $4 \%$ and $31 \%$ (mean change, $18 \%$ ).

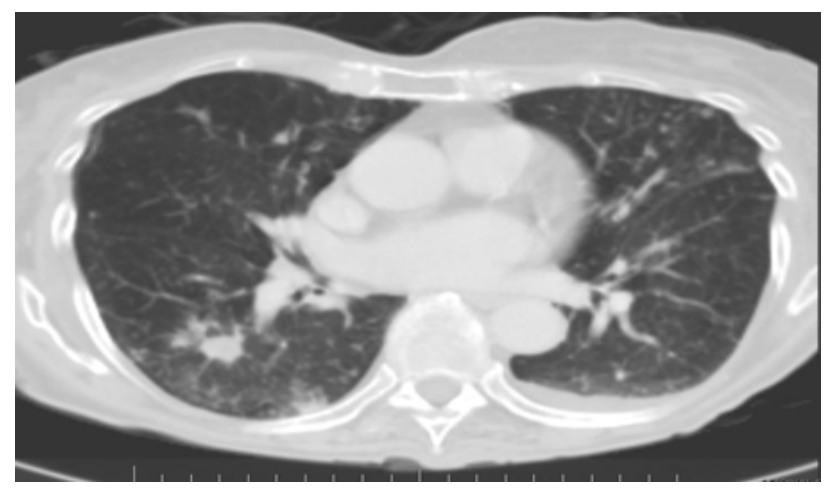

Fig. (2). Computer tomography scan of chest from an 84-year-old woman with one-year history of cough and dyspnea. Note multiple peripheral nodules and an ill-defined pleural-based density in the superior segment of the right lower lobe and several small nodules in the periphery of the left lower lobe.

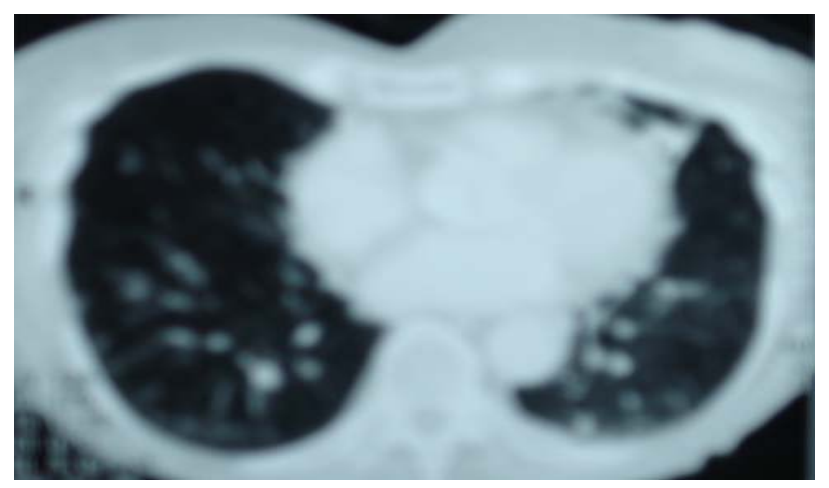

Fig. (3). Computer tomography scan of chest from a 75-year-old woman with two-year history of productive cough. Note multiple bilateral nodules.

Eight (8) patients had abnormal PF to begin with and these had mean increases (percent predicted values) and $p$ values as follows: FEV1 of 10.29 $\pm 4.72,0.001$; FVC of $10.86 \pm 5.34,0.09$ and FEV1/FVC of7.67 $\pm 3.50,0.42$. Two (2) patients had normal $\mathrm{PF}$ and these had minimal change in FEV1, FVC and FEV1/FVC.

\section{DISCUSSION}

We found that MAC lung infection was more prevalent in our institution, consisting of two inner city community hospitals, than we had believed [10]. Until these studies, we thought that MAC lung disease was less frequent than pulmonary tuberculosis. This was believed because the results of MAC positive cultures were only made available to the patient's PMD and since only the PMD was informed of $\mathrm{MAC}$, he/she was likely to ignore/consider this unimportant unless the PMD was a pulmonolgist If the primary physician was a pulmonologist the pulmonary department was informed about the results. On the other hand, in the case of positive results for MTB the pulmonary department was informed regardless of PMD. During the one year period constituting the first study, there were 15 confirmed cases of active pulmonary tuberculosis, 5 of them in immunocompetent patients; it was a surprise to us to find 21 cases of likely MAC lung infection in nonimmunocompromised persons during the same twelve months. This incidence is about 4 times greater than the incidence of tuberculosis. Moreover, the true incidence may be underestimated, as a significant number of patients with active MAC do not have sputa sent for AFB because of low clinical suspicion and lack of pulmonary consultation. We can only speculate whether these observations can be extrapolated to previous years. We believe our findings apply to other hospitals with similar patient mix; many of our patients present to or attend clinics at a number of hospitals in the borough of Queens.

We believe it is important to note that if a pulmonologist was not directly involved in the patient's care, the possibility of MAC lung disease was not considered The nonpulmonologist PMDs were treating the patients for pneumonia. The pulmonary/infectious disease departments were not initially involved. If the patients were referred by the non-pulmonologist PMDs to the institution pulmonary clinic or private pulmonologist the diagnosis of MAC infection was made. This observation points to the need to educate internists and family physicians about this increasingly diagnosed disease that is capable of causing permanent lung damage and even fatality when untreated. With greater awareness, sputa will be evaluated for AFB and pulmonary consultation called to patients with clinical or radiographic abnormalities consistent with MAC. It has been known that MAC lung infection should be considered in middle- aged and elderly patients, especially those with preexisting lung disease [5]. Emphasis on ethnic or national origin (white, U.S.-born) and smoking status appears less important than formerly thought. The findings that half of our patients were white and U.S. born is nevertheless noteworthy because our hospitals serve a diverse patient population including a majority of foreign-born, black, Asian and Hispanic patients.

Reasons to explain the prevalence of MAC disease in immunocompetent individuals may include reduced immunity to mycobacteria in the population because of the reduced prevalence of MTB infection and Bacillus CalmetteGuerin (BCG) vaccination and greater exposure to nontuberculous mycobacteria, primarily MAC, due to changes in personal hygiene habits from bathing to showering [11, 12]. MAC is present in tap water and becomes aerosolized by showerheads. We have no information about BCG vaccinations or whether our patients showered instead of taking baths; use of hot tubs or gardening, which may be risk factors for MAC infection, were unlikely in this population. There is no documentation in the literature of human-to-human transmission of MAC lung disease. It is believed that all cases result from environmental exposure. Smoking was a risk factor, possibly 
Table 1. PFTs Before and After Anti-MAC Treatment

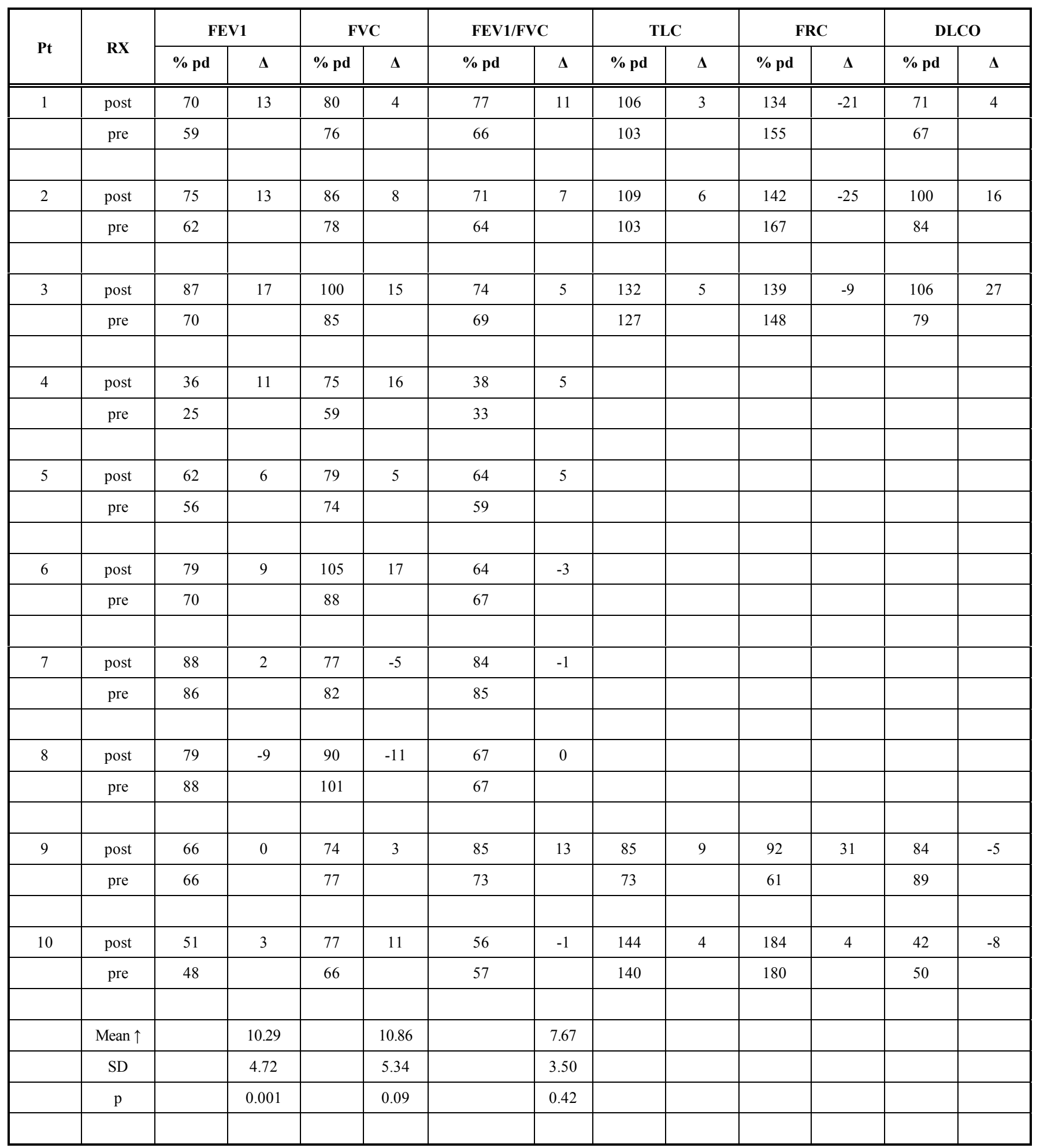

Mean, SD, p values reported for increases in patients with abnormal PFTs.

$\mathrm{Pt}=$ Patient number, RX $=$ Anti-MAC therapy, Post $=$ Post treatment, Pre $=$ Pre-treatmenr, \%pd $=$ Percent predicted, $\Delta=\mathrm{Change}$ from pre and post values, $\uparrow=$ Increase, SD $=$ Standard deviation, $\mathrm{p}=$ Probability.

making the patient more susceptible to infection because of impairment of bronchial clearance mechanisms, underlying lung damage from smoking or the cigarettes serving as a source of MAC.

The 2007 ATS guidelines, as their predecessors, discuss who should be treated for MAC lung disease [7]. The basic requirement is at least 2 positive sputum cultures since a single positive sputum culture may result from environmental contamination. In our experience, however, strict application of this guideline is misleading as two of our patients had only one sputum culture positive for MAC but the clinical presentation and radiographic changes strongly 
supported MAC lung disease. At the other end of the spectrum, one patient had 5 sputum cultures positive. He was treated for community-acquired pneumonia with complete clinical and radiographic resolution by the $9^{\text {th }}$ day. We believe he did not have MAC lung disease and the cultures represented only colonization of his airways. Based on this experience, we strongly believe that clinical-radiographic evidence should prevail over the number of positive cultures when the indications for treatment for MAC are considered culture while another suptum/BAL culture is obtained.

MAC lung disease is more prevalent in this patient population than we previously believed. Greater awareness of this slowly progressive disease is needed. Primary care physicians should be alerted to consider MAC as a possible etiology for chronic lung disease, perhaps through the standard forms used for admission history and diagnosis and for physician orders.

In our study to evaluate how often MAC and MTB coexist we found that 12 patients had combined mycobacterial infection compared with 92 who cultured for MAC alone. Most patients with combined infection were clinically consistent with MTB and responded to anti MTB treatment alone. Most patients were non-white; all were foreign-born in contrast to our experience with MAC infection alone were $50 \%$ of the patients were white and US born.

In our study that evaluated patients who receive antiMAC therapy all eight patients who had abnormal PF showed increases in FEV1, 90\% had an increase in FVC and $80 \%$ had an increase in FEV1/FVC ratio. Three of five patients had an increase in DLCO. The reported differences do not appear to be larger than test variability but were consistent in the same direction. This shows that treatment with anti-MAC therapy improved PF in those patients whose PF was abnormal to begin with.

To the best of our knowledge, these studies were the first to evaluate the prevalence and nature of MAC lung disease and the questions of co-infection with MTB and PF improvements after anti-MAC therapy in inner city community hospitals. We believe similar studies are needed elsewhere to explore the extent to which our findings can be extrapolated to other institutions, geographic areas, and patient population groups.

\section{ETHICS}

These retrospective studies were approved by the Institutional Research Board.

\section{REFERENCES}

[1] Eaton T, Fakinham JO III, Von Reyn CF. Recovery of Mycobacterium avium from Cigarettes. J Clin Microbiol 1995; 33: 2757-58.

[2] Field S, Cowie R. Lung disease due to the more common non tuberculous Mycobacteria. Chest 2006; 129: 1653-72.

[3] Crow HE, King CT, Smith CE, et al. A limited clinical, pathological and epidemiologic study of patients with pulmonary lesions associated with atypical acid - fast bacilli in the sputum. Am Rev Respir Dis 1957; 75: 199-222.

[4] Prince DS, Peterson DD, Steiner RM, et al. Infection with Mycobacterium avium complex in patients without predisposing conditions. N Engl J Med 1989; 321: 863-68.

[5] Beck F. Pulmonary disease due to atypical tubercle bacilli. Am Rev Respir Dis 1959; 80: 738-43.

[6] Warring Fc Jr. Mycobacteria in a New England hospital: a study of mycobacterial species occurring in the sputum of patients with chronic pulmonary disease. Am Rev Respir Dis 1968; 98: 965-77.

[7] American Thoracic Society. Diagnosis, treatment, and prevention of nontuberculous mycobacterial disease. Am J Respir Crit Care Med 2007; 175: 367-416.

[8] Miller A, Thornton JC, Warsaw R, Bernstein J, Teirstein AS, Selikoff IJ. Mean and instantaneous expiratory flows, FVC and FEV1: prediction equations for non-smokers and smokers from a random sample of Michigan, a large industrial state. Bull Physiopath Respir 1986; 22: 589-97.

[9] Miller A, Thornton JC, Warshaw R, Anderson H, Teistein AS, Selikoff IJ. Single breath diffusing capacity in a representative sample of the population of Michigan, a large industrial state: predicted values, lower limits of normal, and frequencies of abnormality by smoking history. Am Rev Respir Dis 1983; 127 : 270-7.

[10] Billinger ME, Olivier KN, Viboud C, et al. Nontuberculous Mycobacteria-associatedl lung disease, in hospitalized persons, United States, 1998-2005. Emerg Infect Dis 2009; 15(10): 1562-9.

[11] Trnka L, Dankova D, Svandova E. Six years experience with the discontinuation of BCG vaccination: Protective effect of BCG vaccination against the Mycobacterium avium intracellulare complex. Tuberc Lung Dis 1994; 75: 348-52.

[12] O'Brien DP, Currie BJ, Krause VL, et al. Non-tuberculous mycobacterial disease in Northern Australia: a case series and review of the literature. Clin Infect Dis 2000; 31: 958-68. 